\title{
The physics and chemistry of circumstellar envelopes of S-stars on the AGB
}

\author{
Sofia Ramstedt ${ }^{1}$, Fredrik L. Schöier ${ }^{2}$, and Hans Olofsson ${ }^{1,2}$ \\ ${ }^{1}$ Dept. of Astronomy, Stockholm University, \\ Roslagstullsbacken 21, SE-10691, Stockholm, Sweden \\ email: sofia@astro.su.se \\ ${ }^{2}$ Onsala Space Observatory
}

\begin{abstract}
Presented here are the preliminary results of a long-term study of S-stars on the AGB. S-stars are important as possible transition objects between oxygen-rich M-stars and carbon stars. The aim of the study is to compare results from our newly gathered observational database for the S-stars with those already obtained for the M- and carbon stars. We can thus follow the changes as the stars evolve along the AGB and more firmly establish the suggested M-MS-S-SC$\mathrm{C}$ evolutionary sequence. It will also allow us to determine the relative importance of processes such as non-equilibrium chemistry, grain formation, and photodissociation in regulating the chemistry in circumstellar envelopes of AGB stars.
\end{abstract}

Keywords. Stars: AGB and post-AGB, (stars:) circumstellar matter, stars: mass loss, stars: abundances

\section{Observational database}

The sample consists of 42 S-type AGB stars, and it is flux-limited. The CO data has been gathered using the Onsala $20 \mathrm{~m}$ telescope $(J=1 \rightarrow 0)$, the IRAM $30 \mathrm{~m}$ telescope $(J=1 \rightarrow 0,2 \rightarrow 1)$, the $\operatorname{JCMT}(J=3 \rightarrow 2)$, and $\operatorname{APEX}(J=3 \rightarrow 2)$. The SiO data has been gathered using the Onsala $20 \mathrm{~m}(J=2 \rightarrow 1, \mathrm{v}=0, \mathrm{v}=1)$, the IRAM $30 \mathrm{~m}(J=2 \rightarrow 1$, $5 \rightarrow 4)$, the $\operatorname{JCMT}(J=6 \rightarrow 5,8 \rightarrow 7)$, and $\operatorname{APEX}(J=8 \rightarrow 7)$. Further HCN data has been gathered using the IRAM $30 \mathrm{~m}(J=1 \rightarrow 0,3 \rightarrow 2)$, the $\operatorname{JCMT}(J=3 \rightarrow 2)$, and $\operatorname{APEX}(J=4 \rightarrow 3)$. Emission from other molecules (CS, SiS) has been searched for, and detected in some of the sample sources.

\section{Radiative transfer modelling}

The circumstellar envelopes (CSEs) are assumed to be spherically symmetric and formed by constant mass-loss rates. Mass-loss rates and physical properties of the CSEs are estimated from the $\mathrm{CO}$ data, using a non-LTE, non-local radiative transfer code based on the Monte Carlo method (Schöier \& Olofsson 2001, Olofsson et al. 2002, Ramstedt et al. 2006). The energy balance is solved self-consistently and the effects of dust on the radiation field and the thermal balance are included. The molecular excitation analysis also includes the radiation field generated by the central star and reprocessed by thermal dust grains in the envelope. Once the physical properties are determined, abundances of the other molecules observed in the CSEs can be estimated using the same radiative transfer code.

\section{Results and conclusions}

- We have estimated the fractional abundance of $\mathrm{SiO}$ in $27 \mathrm{~S}$-stars and find that the abundances can be more than an order of magnitude larger than predicted by thermal equilibrium chemistry (see Figure 1). For a certain mass-loss rate the circumstellar $\mathrm{SiO}$ abundance seems independent of the $\mathrm{C} / \mathrm{O}$-ratio. 
- The previous analysis of M-stars (González Delagado et al. 2003) and carbon stars (Schöier et al. 2006) shows a clear trend that the $\mathrm{SiO}$ abundance decreases as the density of the wind increases. Eventhough the high-mass-loss-rate S-stars are sparse, we see indications of the same trend in this analysis. The trend is indicative of adsorption of $\mathrm{SiO}$ onto grains.

- We have started the modelling of the HCN emission to determine fractional abundances in the S-stars. The results will be compared to the results for the M- and carbon stars (see contribution by Schöier \& Olofsson in these proceedings).

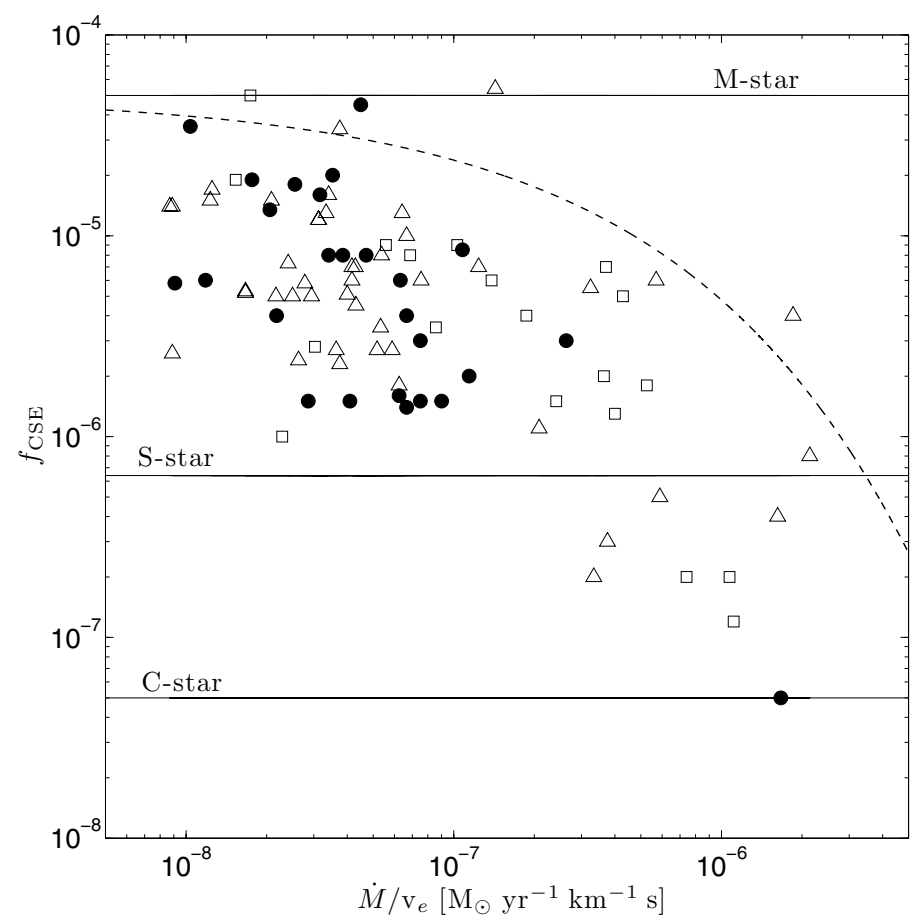

Figure 1. $\mathrm{SiO}$ fractional abundances $\left(f_{\mathrm{CSE}}\right)$, obtained from the radiative transfer modelling, as a function of a density measure for M-stars (open triangles), carbon stars (open squares) and S-stars (filled dots). The horizontal lines mark the abundances predicted by equilibrium chemistry calculations. The dashed line shows the expected $f(\infty)$ (scaled to $5 \times 10^{-5}$, roughly the expected fractional abundance from stellar equilibrium chemistry when $\mathrm{C} / \mathrm{O}<1$ ) for a model including adsorption of $\mathrm{SiO}$ onto dust grains.

\section{Acknowledgements}

The authors acknowledge support from the Swedish Research Council. This work has benifitted from research funding from the European Community's sixth Framework Programme under RadioNet R113CT 20035058187.

\section{References}

González Delgado, D., Olofsson, H., \& Kerschbaum, F. 2003, A\&A, 411, 123

Olofsson, H., González Delgado, D., Kerschbaum, F., \& Schöier, F. L. 2002, A\&̛A, 391, 1053

Ramstedt, S., Schöier, F. L., Olofsson, H., \& Lundgren, A. A. 2006, A\&A (Letters), 454, L103

Schöier, F. L. \& Olofsson, H. 2001, A\& A, 368, 969

Schöier, F. L, Olofsson, H., \& Lundgren, A. A. 2006, A\&GA, 454, 247 